\title{
Étude documentaire
}

Belgique, Danemark, Égypte, Espagne, États-Unis, Pologne

Bernadette Plumelle, Cécile de Bouttemont, Prunelle Charvet, Marion Latour et Sophie Condat

\section{CpenEdition}

Journals

Édition électronique

URL : http://journals.openedition.org/ries/1532

DOI : $10.4000 /$ ries. 1532

ISSN : 2261-4265

Éditeur

Centre international d'études pédagogiques

Édition imprimée

Date de publication : 1 septembre 2004

Pagination : 145-157

ISBN : 978-2-85420-561-8

ISSN : $1254-4590$

Référence électronique

Bernadette Plumelle, Cécile de Bouttemont, Prunelle Charvet, Marion Latour et Sophie Condat, «Étude documentaire ", Revue internationale d'éducation de Sèvres [En ligne], 36 | septembre 2004, mis en ligne le 11 avril 2012, consulté le 01 mai 2019. URL : http://journals.openedition.org/ries/1532 ; DOI :

10.4000/ries. 1532 


\title{
Étude documentaire
}

\author{
Belgique, Danemark, Égypte, Espagne, États-Unis, Pologne
}

\section{Bernadette Plumelle}

Le Centre de ressources documentaires du CIEP a été sollicité pour produire une étude documentaire sur le thème "École et religion» dans quelques pays. Ce dossier, sous forme de tableau, concerne trois domaines : les dispositions constitutionnelles régissant le lien entre l'État et la religion; les structures éducatives publiques et privées; le volume horaire et les contenus de l'enseignement religieux. À l'exception du tableau sur l'Égypte, les informations sont issues des mêmes sources et permettent une lecture comparative.

\section{État et Religion}

Pour définir la nature de la relation entre l'État et l'Église, la classification proposée par Olivier-Louis Séguy («Laïcité et liberté religieuse en Europe», Ateliers, n 17, 1998) a été reprise : «régime séparatiste» pour les États qui ne souhaitent pas entretenir de rapports particuliers avec les autorités spirituelles; «régime confessionnel» pour les pays qui ont choisi de reconnaître leur religion dominante; "régime mixte» pour les États dans lesquels la séparation de l'Église et de l'État se trouve proclamée sans pour autant qu'une relation privilégiée avec une Église ne soit abandonnée. Les textes législatifs et les articles de la Constitution mentionnant le lien entre le pays et la religion sont rappelés.

\section{Structures éducatives}

Cette rubrique apporte des données quantitatives sur les structures éducatives publiques et privées, sachant que les configurations sont variables selon les pays. Les chiffres cités pour les pays de l'Union européenne et pour les États-Unis sont extraits du rapport de l'OCDE Regards sur l'éducation 2003 : les indicateurs de l'OCDE.

\section{Enseignement religieux}

Cette rubrique porte sur trois points : le volume horaire dédié à l'enseignement religieux selon le niveau d'enseignement, les contenus et les modalités d'application. Concernant les pays de l'Union européenne, la première partie reprend les chiffres publiés par la Commission européenne dans Les chiffres clés de l'éducation en Europe.

\section{Sources}

Pour chaque pays, les sources institutionnelles et les références d'ouvrages ou d'articles spécifiques au pays sont rappelées en fin de tableau. Mais un certain nombre de documents utilisés concernent l'ensemble des pays étudiés :

WILLAIME Jean-Paul coord., "École et culture religieuse» in Revue française de pédagogie, $\mathrm{n}^{\circ} 125$, décembre 1998, pp. 5-41, tabl., graph., bibliogr.

L'enseignement privé dans l'Union européenne : organisation administration et rôle des pouvoirs publics, Eurydice/Bruxelles, 2000, 141 p.

EURYDICE / EUROSTAT, Les chiffres clés de l'éducation en Europe, Eurydice/Bruxelles, 2002, $262 \mathrm{p}$.

OCDE, Regards sur l'éducation 2003 : les indicateurs de l'OCDE, OCDE/Paris, 2003.

L'enseignement du fait religieux, Actes du séminaire national interdisciplinaire organisé à Paris les 5, 6 et 7 novembre 2002, CRDP/Versailles, (Les Actes de la Desco), 2003, 375 p.

Juin 2004 


\section{Belgique}

\section{État / Religion}

Régime mixte.

La Constitution de 1831 garantit la liberté religieuse et l'indépendance des cultes vis-à-vis de l'État. Les traitements et pensions des ministres des six cultes reconnus - catholique, protestant, juif, anglican, islamique, orthodoxe sont pris en charge par les pouvoirs publics. Le catholicisme perd son statut de religion officielle. (Le Monde, 12/12/2003).

Article $24-\S 1^{\text {er }}$ de la Constitution : «L'enseignement est libre; toute mesure préventive est interdite; la répression des délits n'est réglée que par la loi ou le décret. La communauté assure le libre choix des parents.»

Le décret du 31 mars 1994, définissant la neutralité dans l'enseignement public, subordonne l'exercice de la liberté religieuse au respect d'autres principes généraux.

\section{Structures éducatives}

Dans l'article 24 de la Constitution $\$ 5$, «l'organisation, la reconnaissance ou le subventionnement de l'enseignement par la communauté sont réglés par la loi ou le décret.»

L'enseignement organisé par les pouvoirs publics est appelé «enseignement officiel»; l'enseignement organisé par des personnes privées est appelé «enseignement libre». Dans ce cas, les écoles sont de deux types : elles sont dites «confessionnelles», essentiellement catholiques, ou "indépendantes». En vertu du Pacte scolaire (loi du 29 mai 1959), l'égalité entre les différents réseaux d'enseignement est garantie; l'accès à l'enseignement préprimaire, primaire et secondaire est gratuit dans les établissements de l'État et dans les établissements subventionnés par l'État. (Source : EURYDICE).

Pourcentage des élèves scolarisés :

- pour l'enseignement primaire : établissements publics : 45,6\%; établissements privés subventionnés par l'État : $54,4 \%$;

- pour le premier cycle de l'enseignement secondaire: établissements publics: 43,1\% ; établissements privés subventionnés par l'État: 56,9\%;

- pour le deuxième cycle de l'enseignement secondaire: établissements publics : 41,6\% ; établissements privés subventionnés par l'État: 58,4\%.

\section{Enseignement religieux}

La définition en est donnée dans l'article 24 de la Constitution:

$\$ 1$. «La communauté organise un enseignement qui est neutre. La neutralité implique notamment le respect des conceptions philosophiques, idéologiques ou religieuses des parents et des élèves. Les écoles organisées par les pouvoirs publics offrent, jusqu'à la fin de l'obligation scolaire, le choix entre 
l'enseignement d'une des religions reconnues et celui de la morale non confessionnelle. »

$\$ 3$. «Tous les élèves soumis à l'obligation scolaire ont droit, à charge de la communauté, à une éducation morale ou religieuse ».

Volume horaire :

- cours de religion/morale pour l'enseignement primaire : $7 \%$ pour la Belgique francophone, 7,1\% pour la Belgique germanophone et flamande;

- cours de religion/morale pour l'enseignement secondaire : 7\% pour la Belgique francophone, 6,8\% pour la Belgique germanophone et 4,8\% pour la Belgique flamande.

\section{Sources}

Textes constitutionnels consultables sur le site du Sénat de Belgique:

http://www.senate.be/

RESTODE, serveur pédagogique de l'enseignement organisé par la Communauté française http://www.restode.cfwb.bel

Données OCDE, 2001.

Données Eurydice, année scolaire 2000/2001.

Bernadette Plumelle

Juin 2004 


\section{Danemark}

\section{État / Religion}

Régime confessionnel.

Article 4 de la Constitution du 5 juin 1953: «l'Église évangélique luthérienne est l'Église nationale danoise et jouit, comme telle, du soutien de l'État". Les dispositions de l'article 4 sont complétées par celles des articles 66 et 69 , qui prévoient que le régime de l'Église nationale, ainsi que celui des autres Églises, sont fixées par la loi.

Article $6:$ :Le roi doit appartenir à l'Église évangélique luthérienne.» Il n'existe pas d'appartenance de plein droit; le baptême est nécessaire pour appartenir à cette Église et il définit une identité culturelle. Les membres du clergé ont un statut de fonctionnaires. La liberté de religion pour les autres cultes est affirmée.

\section{Structures éducatives}

Pourcentage des élèves scolarisés:

- pour l'enseignement primaire : établissements publics : 89,2\% ; établissements privés subventionnés par l'État: 10,8\%;

- pour le premier cycle de l'enseignement secondaire: établissements publics: 87,4\% ; établissements privés subventionnés par l'État: 12,6\%;

- pour le deuxième cycle de l'enseignement secondaire: établissements publics: 95,6\% ; établissements privés subventionnés par l’état: 4,4\%.

Les écoles privées sont des établissements autonomes et indépendants dont les statuts ont été approuvés par le ministère de l'Éducation. Tous les établissements privés sont habilités à recevoir des subsides de l'État pour 80 à $85 \%$ de leurs frais de fonctionnement, sur la base du nombre d'élèves inscrits au sein de l'établissement au cours d'une année donnée.

Il existe des établissements religieux ou confessionnels au sein du secteur privé établissements catholiques ou de la mission danoise et quelques écoles musulmanes.

\section{Enseignement religieux}

Volume horaire :

- cours de religion/morale pour l'enseignement primaire : 5,6\%;

- cours de religion/morale pour l'enseignement secondaire : $4,3 \%$.

L'enseignement religieux n'est pas évoqué dans la Constitution. La religion constitue une matière d'enseignement à tous les niveaux du système scolaire.

Les programmes de l'enseignement religieux sont sous le contrôle du Parlement et non de l'Église et sont le produit de la coopération entre le ministère de l'Éducation nationale et l'association des enseignants. Cet enseignement 
propose dans les écoles secondaires un véritable cours d'histoire des religions dont le programme comprend des leçons sur les religions primitives, sur une ou deux religions non chrétiennes, sur le christianisme et sur les conceptions religieuses et non religieuses de la vie (Le Monde 12/12/03, p. 8).

\section{Sources}

Extrait de l'étude produite par la Division des études de législation comparée du Sénat sur le «Financement des communautés religieuses» (septembre 2001) consultable sur le site du Sénat.

Données OCDE, 2001.

Données Eurydice, année scolaire 2000/2001.

Cécile de Bouttemont

Juin 2004 


\section{Égypte}

\section{État / Religion}

Régime confessionnel.

Constitution de 1971 de la République arabe d'Égypte.

Article 2 : «L'islam est la religion de l'État dont la langue officielle est l'arabe; les principes de la loi islamique constituent la source principale de législation.»

Article 12 : «La société s'engage à sauvegarder la morale, à la protéger et à raffermir les authentiques traditions égyptiennes. Elle doit veiller au maintien du niveau élevé de l'éducation religieuse, des valeurs morales et patriotiques, du patrimoine historique du peuple, des réalités scientifiques, du comportement socialiste et des mœurs publiques, dans les limites de la loi.»

Environ $90 \%$ des Égyptiens sont musulmans sunnites et $9 \%$ sont coptes (chrétiens). Bien que l'État garantisse la liberté de croyance et de culte, tout prosélytisme pour une religion autre que l'islam est interdit.

\section{Structures éducatives}

Depuis le nouveau régime en 1952, l'école est obligatoire et gratuite pour les enfants âgés de six à douze ans. L'enseignement de base (primaire et préparatoire) dure neuf ans selon l'article 4 de la loi de l'enseignement. Au secondaire, les élèves peuvent choisir de suivre des cours d'enseignement général ou technique. On compte trois grands types d'établissements :

- les établissements civils publics (gratuits) accueillent $95 \%$ des élèves ;

- les établissements civils privés appelés «écoles de langue» parce qu'ils dispensent l'enseignement dans une langue étrangère (souvent l'anglais) sont payants; certains proposent, au lieu du baccalauréat égyptien, l'IGCSE (International General Certificate of Secondary Education), son équivalent anglosaxon;

- les écoles azharies (héritières modernes des écoles coraniques) dépendent de l'université islamique El Azhar et ne préparent qu'au baccalauréat azhari qui permet d'accéder uniquement à l'université El Azhar.

\section{Enseignement religieux}

L'article 19 de la Constitution de 1971 stipule que «l'éducation religieuse est une matière essentielle dans les programmes de l'enseignement général. »

La religion musulmane et la religion chrétienne sont enseignées dans les écoles égyptiennes civiles selon des programmes officiels gouvernementaux. 
Les parents ne peuvent ni dispenser leurs enfants d'enseignement religieux, ni choisir leur religion.

Volume horaire :

- cycle primaire: trois heures par semaine sur un total de 27 à 34 heures d'étude ;

- cycle préparatoire et secondaire: 2 heures/semaine sur un total de 34 à 39 heures d'étude.

Les écoles azharies utilisent les manuels du ministère mais fournissent des ouvrages supplémentaires pour les études religieuses. Chaque journée commence par deux heures de Coran. À la fin du cycle primaire, les élèves doivent avoir mémorisé tout le Coran.

\section{Sources}

Textes constitutionnels : http://portail.droit.francophonie.org/doc/html/eg/con/1980dfegcolhtml

Site de Jacques Leclerc de l'université de Laval au Canada sur l'aménagement linguistique et les langues dans le monde : http://www.tffq.ulaval.ca/ax//afrique/egypte.htm

Site de l'ambassade de France en Égypte : http://www.ambafrance-eg.org/pages/cse.html

MSN Encarta : http://fr.encarta.msn.com/encyclopedia_761557408/\%c3\%89gypte.html

Données mondiales de l'éducation/Bureau International d'Éducation (BIE) UNESCO : consultables sur le site du BIE : http://www.ibe.unesco.org

"Egypt's national education debate»/COOK Bradley James; Comparative education, novembre 2000, vol. 36, nº 4, pp. 477-490.

«L'école en Égypte : la modernité reste un combat»/KHOURI-DAGHER Nadia, Le Monde de l'éducation, juin 1996, n²38, pp. 49-54.

Rapport d'information 387 (2002-2003) de la Commission des Affaires étrangères du Sénat: http://www.senat.fr/rap/r02-387/r02-387_mono.html\#toc53

Prunelle Charvet

Juin 2004 


\section{Espagne}

\section{État / Religion}

Régime mixte.

Dans l'article 16 de la constitution du 27 décembre 1978 il est stipulé que l'Espagne est «un État de droit social et démocratique où aucune confession n'aura le caractère de religion étatique»; les pouvoirs publics «tiendront compte des croyances religieuses de la société espagnole et entretiendront de ce fait des relations de coopération avec l'Église catholique et les autres confessions».

La Loi organique sur la liberté religieuse de 1980 distingue les communautés religieuses qui ont un «enracinement notoire en Espagne» et les groupes religieux d'implantation récente. Le critère retenu n'est pas quantitatif, c'est l'ancienneté et l'importance culturelle qui dominent. L'État a ainsi reconnu les minorités protestante, juive et musulmane le 14 décembre 1984 pour les deux premières, le 14 juillet 1989 pour la troisième. (Le Monde 12/12/2003 p. 8).

\section{Structures éducatives}

Pourcentage des élèves scolarisés:

- pour l'enseignement primaire: établissements publics: 66,6\%; établissements privés subventionnés par l'État: 30,2\% ; établissements privés indépendants: $3,2 \%$;

- pour le premier cycle de l'enseignement secondaire : établissements publics : 66,5\% ; établissements privés subventionnés par l'État : $30,3 \%$; établissements privés indépendants : $3,2 \%$;

- pour le deuxième cycle de l'enseignement secondaire: établissements publics : $78,3 \%$; établissements privés subventionnés par l'État : $10,5 \%$; établissements privés indépendants : $11,2 \%$.

L'article 27 de la Constitution «reconnaît aux personnes physiques et juridiques la liberté de créer des centres d'enseignement, dans le respect des principes constitutionnels».

\section{Enseignement religieux}

Volume horaire :

- cours de religion/morale pour l'enseignement primaire: 6,5\%;

- cours de religion/morale pour l'enseignement secondaire: 5,8\%.

L'enseignement catholique doit obligatoirement être assuré par les établissements scolaires pour les élèves souhaitant le suivre. C'est une matière évaluée comme les autres. Les cours de religion sont assurés par du personnel choisi par l'Église et payé par l'État. Il existe néanmoins une alternative: l'histoire des religions ou "fait religieux», enseignée par des professeurs 
d'histoire et de philosophie (approche historico-culturelle). Les élèves de cette option devraient aborder les trois religions monothéistes et des thèmes croisés, tels que politique et religion. Contrairement aux notes attribuées dans l'enseignement religieux catholique, les notes de ces matières alternatives n'entrent pas dans le calcul de la note moyenne de l'année scolaire.

La Cour constitutionnelle espagnole a estimé que la neutralité qui devait caractériser l'enseignement public n'empêchait pas «les établissements publics d'organiser un enseignement religieux facultatif, pour permettre aux parents d'exercer leur droit de choisir, pour leurs enfants, une éducation religieuse et morale en accord avec leurs convictions» (arrêt du 13 février 1981).

L'enseignement religieux pour les groupes protestants, juifs et musulmans est également possible mais uniquement pour ceux ayant passé un accord de coopération avec l'État. En 1996, des conventions pour l'enseignement religieux protestant et l'enseignement islamique ont été signées.

En 1997-1998, 86,64\% des élèves de l'école primaire et 61,69\% des élèves du secondaire suivaient l'enseignement religieux catholique dispensé par 8604 professeurs agréés par les autorités catholiques.

En 1997-1998, 59 professeurs dispensaient des cours de religion protestante dans 156 écoles publiques à 1504 élèves; en 2000, on dénombrait 190 professeurs de religion protestante, surtout dans le primaire. Dans la Communauté autonome de Madrid, l'enseignement religieux islamique a été donné en 1999-2000 à 600 élèves dans six établissements. (J-P Willaime op. cit.).

Le nouveau gouvernement espagnol devrait être amené à prendre position sur la question de l'enseignement religieux dans les prochains mois.

\section{Sources}

Ministerio de educación cultura y deporte (ministère de l'Éducation, de la Culture et du Sport) : http://www.mecd.es/

«L'enseignement religieux dans l'École publique espagnole», Joaquin Mantecon Sancho, Conscience et Liberté, $\mathrm{n}^{\circ}$ 60, 2000, pp. 120-121.

Il existe un texte officiel qui établit les curricula pour l'option confessionnelle catholique correspondant à l'enseignement primaire, à l'enseignement secondaire obligatoire et au Bachillerato et, pour l'école maternelle, correspondant à l'enseignement de la religion catholique. Sont définis pour chaque enseignement les objectifs, contenus et critères d'évaluation. Ces curricula ont été définis par les autorités religieuses compétentes et sont consultables sur http://www.boe.es/.pdf

Données OCDE, 2001.

Données Eurydice, année scolaire 2000/2001. 


\section{États-Unis}

\section{État / Religion}

Régime séparatiste.

Premier amendement de la Constitution en $1791:$ :Le Congrès ne légiférera pas de loi relative à l'établissement d'une religion ou empêchant son libre exercice. Ce droit inaliénable à la liberté religieuse ne dépend ni d'une autorité politique ni d'une élection mais s'enracine dans la dignité inviolable de tout être.»

Les dix premiers amendements de la constitution américaine portent le nom de Bill of rights.

La formule «In God» we trust, initialement inscrite en 1864 sur les billets de banque comme référence unificatrice des États du Nord en guerre contre le Sud, est proclamée en 1965 devise nationale par le président Eisenhower.

\section{Structures éducatives}

Pourcentage des élèves scolarisés:

- pour l'enseignement primaire : établissements publics : 88,4\%; établissements privés indépendants : $11,6 \%$;

154 pour le premier cycle de l'enseignement secondaire : établissements publics : 90,1\% ; établissements privés indépendants : $9,9 \%$;

- pour le deuxième cycle de l'enseignement secondaire : établissements publics : 90,6\% ; établissements privés indépendants : $9,4 \%$;

Distribution des élèves entre 6 et 18 ans par type d'établissement: établissements publics de la circonscription scolaire désignée : 75,9\%; établissements publics choisis par les parents: $14,5 \%$; établissements privés confessionnels : 7,3\% ; établissements privés non confessionnels : 2,3\%. (source: U.S. Department of Education, National Household Education Surveys Program, NHES : «Parent interview» survey, 1999).

\section{Enseignement religieux}

Il est difficile d'avoir une idée du volume horaire moyen dans la mesure où les responsabilités éducatives incombent aux États qui déterminent notamment le curriculum. Néanmoins, le gouvernement édite des principes directeurs pour aider à l'intégration de la religion à l'école. Deux textes clés sont à signaler :

- Guidance on constitutionally protected prayer in public elementary and secondary schools, publié par le ministère de l'Éducation en février 2003;

- Finding common ground: a guide to religions liberty in public schools, Charles C. Haynes et Oliver Thomas, février 2002, publié par le First Amendment Center. Le chapitre 7 stipule que l'étude de la religion peut être soit 
intégrée à certains cours (société, littérature et arts), soit faire l'objet d'un enseignement spécifique (étude des religions du monde, Bible comme texte littéraire ou littérature religieuse en Occident et en Orient).

\section{Sources}

Site du ministère fédéral: U.S. Department of Education, National Household Education Surveys Program, NHES : http://www.ed.gov

Guide sur la religion et l'enseignement public de Charles C. Haynes et Oliver Thomas (février 2002), ouvrage téléchargeable chapitre par chapitre sur: http://www.firstamendmentcenter.org/about.aspx?id=6276

Données OCDE, 2001.

Marion Latour

Juin 2004 


\section{Pologne}

\section{État / Religion}

Régime séparatiste.

Article 53 de la Constitution du 7 avril 1997 :

- «Toute personne a droit à la liberté de conscience et de religion».

- «La liberté de religion implique la liberté d'avoir ou d'adopter la religion de son choix et la liberté de manifester sa religion (...) par le culte, la prière, l'accomplissement des rites, les pratiques et l'enseignement.»

- «Les parents ont le droit d'assurer aux enfants l'éducation et l'enseignement moral et religieux conformément à leurs propres convictions.»

- «La religion d'une Église ou d'une autre union confessionnelle à statut juridique régulier peut être enseignée à l'école, ce qui ne peut porter atteinte à la liberté de conscience et de religion d'autrui.»

- «Nul ne peut subir la contrainte de participer ou de ne pas participer aux pratiques religieuses.»

- «Nul ne peut être engagé par les autorités de la puissance publique à révéler sa conception du monde, ses convictions religieuses ou sa confession.»

\section{Structures éducatives}

Pourcentage des élèves scolarisés:

- pour l'enseignement primaire : établissements publics : 99,1\%; établissements privés subventionnés par l'État : 0,9\%;

- pour le premier cycle de l'enseignement secondaire : établissements publics : 98,9\% ; établissements privés subventionnés par l'État : $1,1 \%$;

- pour le deuxième cycle de l'enseignement secondaire : établissements publics : 93,3\% ; établissements privés subventionnés par l'État: 6,6\%; établissements privés indépendants : $0,1 \%$.

Loi sur l'enseignement du 7 septembre 1991 : possibilité de fonder des écoles privées à tous les degrés d'enseignement. Jusqu'en 1989, les écoles privées étaient interdites. Écoles non publiques : écoles civiques (sociales), écoles de l'Église ou écoles privées. Depuis 1990, les écoles non publiques sous contrat peuvent bénéficier de subventions de l'État allant jusqu'à $50 \%$ des coûts calculés sur la base des coûts moyens de formation dans une école publique. Il existe des écoles privées non subventionnées par l'État (sans contrat). On comptait en 2000-2001 420 écoles élémentaires non publiques (sur 16 766) dont 53 religieuses; 451 collèges (sur 6295) dont 77 religieux; 461 lycées (sur 2292 ) dont 88 religieux; 624 écoles professionnelles (sur 8281 ) et 1639 écoles de niveau post-baccalauréat (sur 2567). 


\section{Enseignement religieux}

Volume horaire :

- cours de religion/morale pour l'enseignement primaire : 7,7\% ;

- cours de religion/morale pour l'enseignement secondaire : 7,3\%.

Loi sur l'enseignement du 7 septembre 1991 :

Article 12.1: «Reconnaissant le droit des parents à l'éducation religieuse des enfants, les écoles publiques d'enseignement primaire organisent l'enseignement du catéchisme sur la demande des parents, les école publiques d'enseignement secondaire - sur les souhaits soit des parents soit des élèves, les élèves ayant 18 ans décident eux-mêmes sur leur apprentissage du catéchisme. »

- Choix entre des cours de religion et de morale.

- Possibilité pour les parents de demander des cours d'éducation religieuse dans n'importe laquelle des religions reconnues. Les cours sont financés par le ministère de l'Éducation nationale.

Concordat signé entre la Pologne et le Vatican le 28 juillet 1993 : Article 12 : «Concernant le contenu de l'enseignement et de l'éducation religieuse, les catéchistes sont soumis à la réglementation de l'Église, et pour le reste des questions à la loi polonaise.»

\section{Sources}

Constitution de la République de Pologne: http://www.accpuf.org/pol/constit.htm

Portail sur la Pologne : http://www.pologne.gov.pl

Le système éducatif polonais et la réforme du système éducatif en Pologne : http://www.olitul.neostrada.p/CECO/pologne/polsysteduc.htm

Données OCDE, 2001.

Eurydice, Complément à l'étude sur les structures des systèmes d'enseignement et de formation initiale dans l'Union européenne.

Données Eurydice, année scolaire 2000/2001.

Second rapport de la Commission européenne sur la Pologne contre le racisme et l'intolérance adopté le 10 décembre 1999.

Sophie Condat

Juin 2004 
